\title{
Interactive binocular
treatment (I-BiT) for \\ Interactive binocular
treatment (I-BiT) for amblyopia: results of a pilot study of 3D shutter glasses system

$$
\text { system }
$$

\begin{abstract}
Purpose A computer-based interactive binocular treatment system (I-BiT) for amblyopia has been developed, which utilises commercially available 3D 'shutter glasses'. The purpose of this pilot study was to report the effect of treatment on visual acuity (VA) in children with amblyopia.
\end{abstract}

Methods Thirty minutes of I-BiT treatment was given once weekly for 6 weeks.

Treatment sessions consisted of playing a computer game and watching a DVD through the I-BiT system. VA was assessed at baseline, mid-treatment, at the end of treatment, and at 4 weeks post treatment. Standard summary statistics and an exploratory one-way analysis of variance (ANOVA) were performed.

Results Ten patients were enrolled with strabismic, anisometropic, or mixed amblyopia. The mean age was 5.4 years. Nine patients $(90 \%)$ completed the full course of I-BiT treatment with a mean improvement of $0.18(S D=0.143)$. Six out of nine patients $(67 \%)$ who completed the treatment showed a clinically significant improvement of 0.125 LogMAR units or more at follow-up. The exploratory one-way ANOVA showed an overall effect over time $(F=7.95, P=0.01)$. No adverse effects were reported.

Conclusion This small, uncontrolled study has shown VA gains with 3 hours of I-BiT treatment. Although it is recognised that this pilot study had significant limitations-it was unblinded, uncontrolled, and too small to permit formal statistical analysis-these results suggest that further investigation of I-BiT treatment is worthwhile.

N Herbison ${ }^{1}$, S Cobb², R Gregson ${ }^{1}$, I Ash ${ }^{1}$, R Eastgate ${ }^{2}$, J Purdy ${ }^{3}$, T Hepburn², D MacKeith', A Foss ${ }^{1,2}$ and the I-BiT study group

Eye (2013) 27, 1077-1083; doi:10.1038/eye.2013.113; published online 28 June 2013

Keywords: amblyopia; binocular; strabismus; anisometropia; virtual reality

\section{Introduction}

Conventional childhood treatment for amblyopia with an eye patch has been shown to be effective. ${ }^{1}$ However, patching treatment can be distressing for the child and have a negative impact on school and family life. ${ }^{2-4}$ Compliance with patching treatment is often poor, resulting in a sub-optimal treatment outcome. ${ }^{5}$ The only alternative to patching that is used with any frequency is penalisation, most commonly with atropine. ${ }^{6}$ Both patching and penalisation have variable efficacy and require long treatment times. ${ }^{7,8}$ There is, therefore, a need for a treatment for amblyopia, which is both effective and tolerable, and ideally has a shorter time to sustained efficacy when compared with conventional treatments.

The interactive binocular treatment (I-BiT) prototype system ${ }^{9}$ was developed as an alternative to patching treatment and utilised a virtual reality technology. The treatment was designed to appeal to children and therefore improve compliance. Computer games and video footage were displayed in such a way that the amblyopic eye of a patient could be preferentially stimulated during binocular viewing conditions. Visual acuity (VA) gains were demonstrated with prototype systems using different display devices including the 'Cyberscope' desk viewer system (results reported in the study by Waddingham et al ${ }^{10}$ ) and the 'Cy-visor' system (results reported in
${ }^{1}$ Nottingham University Hospitals NHS Trust, Nottingham, UK

${ }^{2}$ The University of Nottingham, Nottingham, UK

${ }^{3}$ The University of Bradford, Bradford, UK

Correspondence: $\mathrm{N}$ Herbison, Orthoptic Department, EENT Building, Queen's Medical Centre, Nottingham University Hospitals, Derby Road, Nottingham NG7 2UH, UK. Tel: $+44(0) 115970$ 9750; Fax: + $44(0) 1158754525$. E-mail: Nicola.herbison@ nuh.nhs.uk

Received: 28 October 2012 Accepted in revised form: 1 May 2013 Published online: 28 June 2013 
the study by Cleary et $a l^{11}$ ). This paper reports early results from use of commercially available $3 \mathrm{D}$ shutter glasses with bespoke I-BiT game-based software and video presentation.

\section{Materials and methods}

\section{I-BiT shutter glasses system}

The I-BiT system hardware (see Figure 1) comprises a desktop PC with two monitors, one for the clinician and one for the patient. As with all previous I-BiT system prototypes, the clinician monitor is used to control the treatment the patient receives and the patient monitor displays the visual stimuli. The system uses 3D shutter glasses and their corresponding infra-red emitter. The patient monitor is a flat-screen 18-inch 3D monitor with a refresh rate of $120 \mathrm{~Hz}$ as required for use with the shutter glasses. The shutter glasses' lenses lighten and darken in synchrony with the monitor but faster than the user can perceive. The patient sits on a comfortable gaming beanbag for the duration of the treatment.

This I-BiT system relies on the same principle as previous prototypes. ${ }^{9}$ Images are presented to both eyes but parts of the image are presented only to the amblyopic eye. The visual scene with the I-BiT system is not presented stereoscopically. Instead, the 3D technology is used to present a distinct but visually related image to each eye allowing the perception of a dynamic, two-dimensional visual scene. The I-BiT system can display video footage and interactive games. A gaming control pad is used for the games. The games have been specifically developed to appeal to children aged $\leq 8$ years. The visual stimuli presented in the current study are described below.

\section{Video stimulus}

The principle used is that the image is divided into two zones. There is an outer 'border' termed a locking stimulus, which is presented to both eyes, while the inner part of the screen presents the video footage to the amblyopic eye. Images within the border can be selectively shown to either eye to act as a control to ensure binocular viewing. To increase compliance, the I-BiT system has a built-in DVD player, and this allows children to watch a DVD of their own choice.

\section{Game stimulus}

An interactive game called ' $N u x^{\prime}$ was used to provide the game-play. In Nux, a player moves through a colourful two-dimensional space-like environment. Points are rewarded for collecting coins and shooting enemies and deducted for colliding with enemies and obstacles (eg, asteroids). Through the I-BiT system, the player and the background are shown to both eyes, but the obstacles, enemies, and coins are shown only to the amblyopic eye. Therefore, in order for the children to play the game successfully, they must be using their amblyopic eye. If the patient was unable to play because of dense suppression or severely reduced VA in their amblyopic eye, then the settings were adjusted such that a proportion of the objects (coins etc) were seen by the non-amblyopic eye.

\section{Patient selection}

Patients were recruited from the Orthoptic department at the Queen's Medical Centre site of Nottingham University Hospitals, who met the following eligibility criteria:

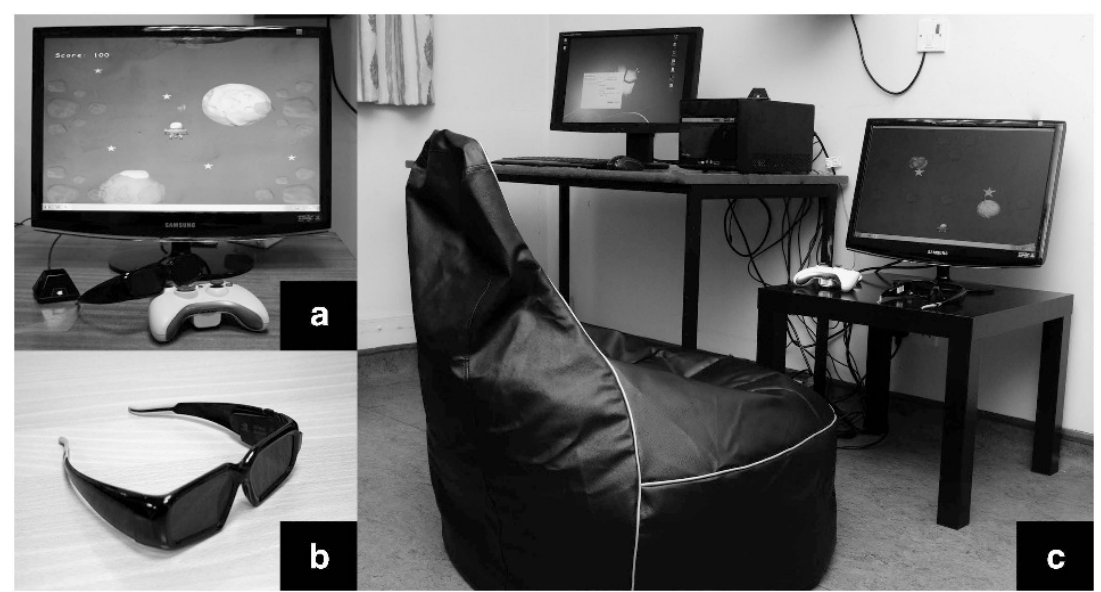

Figure 1 The I-BiT system. (a) 3D monitor and game control pad. (b) Shutter glasses. (c) Complete I-BiT system including clinician and patient monitors. 


\section{Inclusion criteria:}

- Diagnosis of anisometropic, strabismic, or mixed amblyopia as made by an orthoptist (difference of 2 LogMAR units or more using a LogMAR crowded test).

- Aged 4-8 years inclusive.

- Informed consent from legal guardian and assent from patient.

\section{Exclusion criteria:}

- Organic lesions of the eye preventing the establishment of good vision (eg, media opacities, abnormalities in the fundus or optic nerve) and this includes all forms of stimulus deprivation amblyopia.

- Lesions of the brain preventing the establishment of good vision (eg, cortical visual impairment).

- Patients diagnosed with photosensitive epilepsy.

- Loss of suppression at filter $\leq 4$ less as measured with the Sbisa bar.

- Establishment of normal vision by refractive adaptation (wearing glasses after presentation).

- Inability to comply with the follow-up visits required.

- Participation in a previous study examining I-BiT treatment.

\section{Treatment regimen}

All patients underwent cycloplegic refraction and had been wearing their glasses for at least 18 weeks before treatment. Patients attended the orthoptic clinic for $30 \mathrm{~min}$ of I-BiT treatment once a week for 6 weeks, giving a maximal total treatment time of 3 hours. Treatment sessions comprised viewing 20 min of video footage and $10 \mathrm{~min}$ of interactive game-play. Patients returned 4 weeks after their final treatment session for a follow-up assessment. After completion or exit from the study, they remained under the care of the Orthoptic department.

\section{Clinical assessment}

VA was assessed with the LogMAR crowded Keeler test (formally known as the Glasgow acuity test-see McGraw et $a l^{12}$ ) or the LogMAR crowded Kay's picture test. Test selection depended on the ability of the patient, and for each patient the same test was used throughout.

The patients received a full orthoptic assessment and VA assessment at baseline, before the start of the treatment. VA was measured at weeks 3 (mid-treatment), 6 (end of treatment), and 10 (follow-up-4 weeks after the final treatment). For strabismic patients with no demonstrable binocular vision, density of suppression was measured with the Sbisa bar at weeks 3 and 6 . It was decided that patients with a Sbisa bar result of ' $\leq 4$ ' would be withdrawn from the study because of the risk of diplopia.

\section{Outcome measures and statistical analysis}

The primary outcome measure was VA. Secondary measures were compliance and safety.

VA was measured at baseline and then at weeks 3, 6, and 10 (follow-up). Changes from baseline to each posttreatment time point were calculated. An improvement of $\geq 0.125$ LogMAR units was considered clinically significant. ${ }^{12}$ In addition, the proportion of change was calculated at weeks 6 and at follow-up as follows (using the formula described by Stewart et al ${ }^{13}$ ):

Proportion of change $=\mathrm{VA}_{\mathrm{as}}-\mathrm{VA}_{\mathrm{ae}} / \mathrm{VA}_{\mathrm{as}}-\mathrm{VA}_{\mathrm{fe}}$ where $\mathrm{VA}_{\mathrm{as}}$ is the vision of amblyopic eye at baseline, $\mathrm{VA}_{\mathrm{ae}}$ is the vision of amblyopic eye at the end of the treatment (ie, weeks 6 and follow-up), and $\mathrm{VA}_{\mathrm{fe}}$ is the vision of the non-amblyopic eye at the end of treatment.

Compliance was measured as the proportion of planned treatment visits completed by each patient. Safety was measured as the number and type of adverse events reported.

The data were examined using standard summary statistics and included, where appropriate, 95\% confidence intervals. A one-way analysis of variance (ANOVA) was performed on the VA data to determine whether there appeared to be an effect of measurement time on VA, and pairwise comparisons of means were then performed, examining the changes from baseline to 3 and 6 weeks after treatment start and from baseline to the follow-up visit. Statistical analyses were performed using SPSS Version 18 (SPSS Inc., Chicago, IL, USA) and were considered exploratory in nature.

No subgroup analyses were performed.

The study was not statistically powered as it was a pilot study. It was planned that 10 patients would be enrolled and that this would be sufficient to determine whether there was a notable effect using I-BiT and to provide an indication of the magnitude and variability of any effect, which could be used to inform further development.

\section{Statement of ethics}

All applicable institutional and governmental regulations concerning the ethical use of human volunteers were followed during this research. 


\section{Results}

\section{Patient characteristics}

Ten consecutive patients who fulfilled the inclusion/ exclusion criteria were enrolled between August and October 2010. The mean age was 65 months (5.4 years). There were five boys and five girls. Three patients had strabismic amblyopia, four had anisometropic amblyopia and three had mixed (strabismic and anisometropic) amblyopia. Three patients had undergone previous treatment with occlusion, whereas the others had not undergone any previous treatment for amblyopia (other than glasses).

Of these patients, one male patient (aged 4.4 years) was withdrawn from the study after three of the planned six treatments because of a lack of compliance with the treatment-he was restless and easily lost concentration. The remaining nine patients completed all treatment visits for the study. Two patients who completed the treatment regime attended the planned 4-week posttreatment visit at weeks 13 and 15. Their data were included for all summaries of follow-up data.

\section{Visual acuity}

The VA for each patient is shown in Table 1 and summarised in Figure 2. By the end of the 6-week treatment period, all nine patients who had completed treatment showed an improvement in VA. These improvements ranged from 0.025 to 0.45 LogMAR units with a mean of $0.18(S D=0.143)$ and a median of 0.175 . By week 10 (4 weeks post treatment), four out of nine patients remained stable or showed further improvement in VA, and five out of nine patients showed some deterioration (see Table 1).

Overall, from baseline to follow-up, seven of the nine patients $(78 \%)$ showed an improvement. Six of the nine patients $(67 \%)$ demonstrated a clinically significant increase in VA of $\geq 0.125$, ranging from 0.175 to 0.300 LogMAR units. The mean change from baseline to follow-up was $0.13(\mathrm{SD}=0.14)$ with a median of 0.175 .

The exploratory one-way ANOVA showed an overall effect over time $(F=7.95, P=0.01)$ with the subsequent pairwise comparisons indicating an effect between baseline and week 6 .

The mean changes from baseline to weeks 3, 6, and follow-up with corresponding $95 \%$ confidence intervals are shown in Figure 3.

\section{Proportional change in VA}

The mean proportional change in VA at 6 weeks was $32.2 \%(\mathrm{SD}=21.2 \%)$ with a median of $25 \%$ and a range of $9-67 \%$ based on the nine patients who completed the treatment. The mean proportional change in VA (from baseline) at follow-up was $20.1 \%(\mathrm{SD}=27.5 \%)$ with a median of $28 \%$ and a range of -40 to $44 \%$.

\section{Compliance}

Nine of the 10 patients (90\%) attended all treatment sessions. Two of these nine patients did not attend their follow-up visit at week 10, although they did undergo follow-up assessments later (week 13 and week 15).

Actual treatment time in minutes was recorded at each treatment session for games and video separately. For all 10 patients (including the patient who withdrew at week 3$)$, the mean $( \pm S D)$ total treatment time was 159.3 ( \pm 38.9 ) minutes and overall $88.5 \%$ of the planned treatment was administered including $80.6 \%$ of planned game treatment and $92.3 \%$ of video treatment. Considering all patients over all six treatment visits, the minimum durations of planned game and video play

Table 1 Patient characteristics and visual acuity scores in the amblyopic eye

\begin{tabular}{|c|c|c|c|c|c|c|c|c|}
\hline $\begin{array}{l}\text { Patient } \\
\text { Number }\end{array}$ & $\begin{array}{l}\text { Type of } \\
\text { amblyopia }\end{array}$ & $\begin{array}{l}\text { Previous } \\
\text { occlusion }\end{array}$ & Baseline & Week 3 & Week 6 & $\begin{array}{c}\text { Change in } V A^{\mathrm{a}} \\
\text { (baseline to week 6) }\end{array}$ & $\begin{array}{c}\text { Week } 10 \\
(\text { follow-up })\end{array}$ & $\begin{array}{c}\text { Sustained change } \\
\text { in VA } \\
\text { (baseline to follow-up) }\end{array}$ \\
\hline 1 & Anisometropic & No & 0.450 & 0.425 & 0.350 & 0.100 & 0.300 & 0.150 \\
\hline 2 & Mixed & No & 0.700 & 0.300 & 0.250 & 0.450 & 0.400 & 0.300 \\
\hline 3 & Strabismic & No & 0.875 & 0.825 & - & - & - & - \\
\hline 4 & Anisometropic & No & 0.625 & 0.525 & 0.450 & 0.175 & 0.450 & 0.175 \\
\hline 5 & Strabismic & No & 0.700 & 0.625 & 0.350 & 0.350 & 0.525 & 0.175 \\
\hline 6 & Strabismic & Yes & 0.900 & 0.800 & 0.675 & 0.225 & 0.650 & 0.250 \\
\hline 7 & Anisometropic & No & 0.275 & 0.300 & 0.250 & 0.025 & 0.300 & -0.025 \\
\hline 8 & Anisometropic & No & 0.475 & 0.400 & 0.275 & 0.200 & 0.300 (week 15) & 0.175 \\
\hline 9 & Mixed & Yes & 0.475 & 0.500 & 0.400 & 0.075 & 0.625 (week 13) & -0.150 \\
\hline 10 & Mixed & Yes & 0.575 & 0.575 & 0.525 & 0.050 & 0.475 & 0.100 \\
\hline
\end{tabular}

The columns in bold show the amount by which VA changed following treatment.

${ }^{a}$ Change from baseline to week 6 .

${ }^{\mathrm{b}}$ Change from baseline to follow-up. 


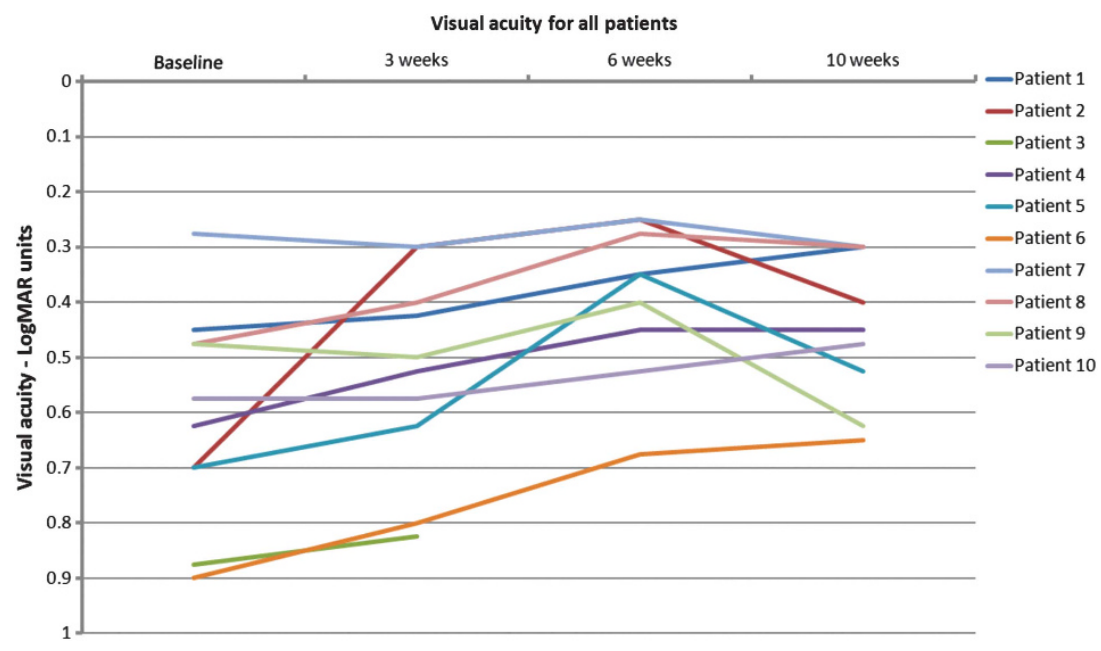

Figure 2 Visual acuity. VA in LogMAR units for all patients from baseline to week 10.

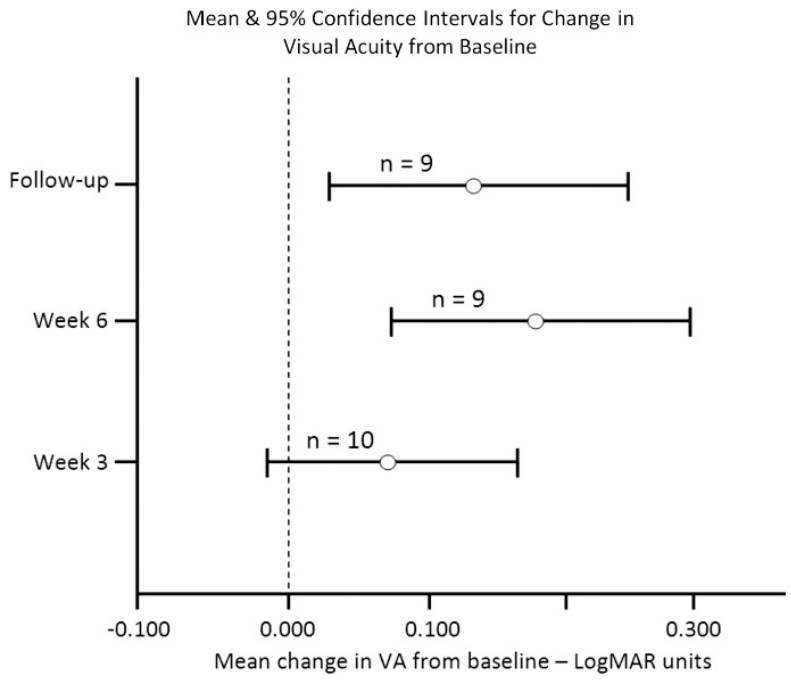

Figure 3 Change in VA. Mean and 95\% confidence intervals for change in VA from baseline.

were 0 and $10 \mathrm{~min}$, respectively, with the maximum being 15 and $30 \mathrm{~min}$, respectively.

\section{Safety}

No adverse reaction was recorded for any patient.

\section{Discussion}

Amblyopia affects $\sim 2 \%$ of children ${ }^{14}$ and accounts for the majority of visits to the paediatric eye clinic. ${ }^{15}$ Existing treatments are lengthy and unpopular with poor compliance being a barrier to treatment success. ${ }^{16}$
The I-BiT system uses computer games and video footage that appeal to children and, therefore, has the potential to improve compliance over traditional patching therapy.

This study found a positive effect of I-BiT treatment on VA, of a magnitude similar to I-BiT systems used in previous studies. ${ }^{10}$ Early I-BiT prototypes were not user-friendly for children and the equipment was too large to be accommodated in the normal clinical environment. This small study has demonstrated that commercially available 3D shutter glasses can be adapted using specialist software to provide effective amblyopia treatment. Ergonomically, this system is superior to former systems and the feedback from patients has been positive.

VA is the primary end-point measure and can be difficult to standardise when testing young children. The LogMAR crowded Keeler test (formerly known as Glasgow Acuity Cards) is a sensitive VA test detecting differences of $0.100 \operatorname{LogMAR}$ units and has a high test-retest reliability. ${ }^{12}$ Therefore, a difference of $>0.100$ LogMAR units, that is, 0.125 , shows a clinically significant change in VA and was chosen as a benchmark of VA change for this study. One of the limitations to this study is that the clinician delivering the treatment was not blind to the VA results. An independent orthoptist assessed VA at weeks 3, 6, and 10 using the same vision test throughout (Crowded LogMAR Keeler Cards or Crowded LogMAR Kay Picture Test). However, a number of different assessors performed the VA assessment, which limited standardisation.

All 10 children attended their weekly appointments throughout the I-BiT treatment and enjoyed the sessions. One of the younger patients found it difficult to concentrate during the $20 \mathrm{~min}$ of video watching and was withdrawn from the study at week 3 . The current 
software does not allow the angle of deviation to be corrected and one of the reasons for his lack of concentration was attributed to the dense suppression of his amblyopic eye due to strabismic amblyopia. We have further adapted the DVD software for future trials to enable the clinician to alter the transparency settings for the non-amblyopic eye. This is to encourage continued viewing and fixation with the amblyopic eye.

This pilot study does have significant limitations. The sample size was too small to permit formal statistical analyses, and the design was not robust. It was uncontrolled and the clinician delivering the treatment was not blind to the VA assessment results. Improvements in VA may have been influenced by bias or a training effect from repeated VA measurements. Furthermore, there were issues in ensuring that all patients received identical treatment. The treatment time was very short compared with conventional treatments and a reduced effect was found at week 10, indicating a possible need to provide treatment over a longer period. However, the results found in this pilot study warrant further investigation of I-BiT with a randomised controlled trial.

In preparation for the randomised controlled trial, the system has been further developed following the results of this pilot study to include DVD transparency settings and extended game-play. The next trial will include independent VA assessments by the same orthoptist to improve standardisation. A control arm has also been introduced that will compare stimulation to the amblyopic eye $v s$ equal stimulation to both eyes (ie, I-BiT vs non I-BiT). For the next study, we will look specifically at analysing any differences between the two different stimuli. This will establish whether DVD viewing or interactive computer game playing produce similar results.

Subgroup analysis was inappropriate in this I-BiT study, but could be planned for a larger trial. Factors that may influence outcome with I-BiT, such as age at presentation or baseline VA, could be investigated in future studies and could help to target treatment effectively. I-BiT could potentially improve the quality of life (QoL) for patients undergoing treatment for amblyopia. It may also reduce treatment duration and, therefore, cost to health-care providers. Future comparisons of I-BiT with conventional treatment could include cost analysis and QoL measures.

The authors acknowledge that the gains in VA are small. However, previous studies have demonstrated that a duration of $120 \mathrm{~h}$ of conventional patching is required to improve VA by 0.100 LogMAR units. ${ }^{1}$ In this study, $67 \%$ of patients improved by $\geq 0.125$ LogMAR units with only $3 \mathrm{~h}$ of treatment time. This reduction in treatment time shows that the I-BiT system has the potential to provide effective amblyopia treatment, which is an acceptable alternative to conventional occlusion.

\section{Summary}

What was known before

- Conventional treatment for amblyopia with an eye patch has a lengthy treatment duration and can be distressing for the child and family.

- Compliance with patching is often poor.

- A virtual reality-based binocular treatment for amblyopia may be more acceptable to children and therefore encourage compliance and improve VA outcome.

- The former I-BiT system prototype produced promising pilot data.

What this study adds

- Advances in virtual reality technology have enabled the development of an I-BiT system, which is ergonomically far superior for both the clinician and the patient.

- Pilot data have revealed VA gains with this latest I-BiT system with a short treatment time.

- Further investigation of this treatment modality is warranted, including a randomised controlled trial and further software development to enable longer gameplay.

\section{Conflict of interest}

The authors declare no conflict of interest.

\section{Acknowledgements}

This study was funded by The Wellcome Trust (Translation Award 090465). We thank Paul Jones (University of Hull) for programming the I-BiT software. Our special thanks to Stephen Haworth for his part in the invention. We also thank all members of the I-BiT steering group for their continued support of the project. The I-BiT project received a Wellcome Trust Translation Award (ref: 090465), which funded this pilot study. The patent for the I-BiT system is held jointly by Nottingham University Hospitals NHS Trust and the University of Nottingham.

\section{References}

1 Stewart C, Moseley M, Stephens D, Fielder A. Treatment dose-response in amblyopia therapy: the Monitored Occlusion Treatment of Amblyopia Study (MOTAS). Invest Ophthalmol Vis Sci 2004; 45: 3048-3054.

2 Koklanis K, Abel LA, Aroni R. Psychosocial impact of amblyopia and its treatment: a multidisciplinary study. Clin Exp Ophthalmol 2006; 34: 743-750.

3 Loudon SE, Passchier J, Chaker L, de Vos S, Fronius M, Harrad RA et al. Psychological causes of non-compliance 
with electronically monitored occlusion therapy for amblyopia. Br J Ophthalmol 2009; 93: 1499-1503.

4 Williams C, Horwood J, Northstone K, Herrick D, Waylen A, Wolke D. ALSPAC Study Group. The timing of patching treatment and a child's wellbeing. Br J Ophthalmol 2006; 90: 670-671.

5 Awan M, Proudlock FA, Gottlob I. A randomized controlled trial of unilateral strabismic and mixed amblyopia using occlusion dose monitors to record compliance. Invest Ophthalmol Vis Sci 2005; 46: 1435-1439.

6 Amenos M, Newsham D. Recent advances in the management of amblyopia. Optometry Practice 2011; 12(3): 119-128.

7 Awan M, Proudlock FA, Grovesnor D, Choudhuri I, Sarvanananthan N, Gottlob I. An audit of the outcome of amblyopia treatment: a retrospective analysis of 322 patients. Br J Ophthalmol 2010; 94: 1007-1011.

8 Simons K, Stein L, Sener EC, Vitale S, Guyton DL. Full-time atropine, intermittent atropine, and optical penalization and binocular outcome in treatment of strabismic amblyopia. Ophthalmology 1997; 104(12): 2143-2155.

9 Eastgate RM, Griffiths GD, Waddingham PE, Moody AD, Butler TKH, Cobb SV et al. Modified virtual reality technology for treatment of amblyopia. Eye 2006; 20: 370-374.
10 Waddingham PE, Butler TKH, Cobb SV, Moody AD, Comaish IF, Haworth SM et al. Preliminary results from the use of the novel Interactive Binocular Treatment (I-BiT) System in the treatment of amblyopia. Eye 2006; 20: 375-378.

11 Cleary M, Moody AD, Buchanan A, Stewart H, Dutton GN. Assessment of a computer-based treatment for older amblyopes: the Glasgow Pilot Study. Eye 2009; 23: 124-131.

12 McGraw PV, Winn B, Gray LS, Elliott DB. Improving the reliability of visual acuity measures in young children. Ophthal Physiol Opt 2000; 20(3): 173-184.

13 Stewart CE, Moseley MJ, Fielder AR. Defining and measuring treatment outcome in unilateral amblyopia. Br J Ophthalmol 2003; 87: 1229-1231.

14 Williams C, Northstone K, Harrad RA, Sparrow JM, Harvey I. ALSPAC Study Team. Amblyopia treatment outcomes after preschool screening $\mathrm{v}$ school entry screening: observational data from a prospective cohort study. Br J Ophthalmol 2003; 87: 988-993.

15 Rahi JS, Logan S, Timms C, Russell-Eggitt I, Taylor D. Risk, causes, and outcomes of visual impairment after loss of vision in the non-amblyopic eye: a population-based study. Lancet 2002; 360(9333): 597-602.

16 Woodruff G, Hiscox F, Thompson JR, Smith LK. Factors affecting the outcome of children treated for amblyopia. Eye (Lond) 1994; 8: 627-631. 